\title{
The usefulness of APIs for new business creation and enterprise innovation
}

\author{
Angel M. Ojeda, Ana G. Méndez University, aojeda5uagm.edu \\ Juan Valera, Ana G. Méndez University, valeraj1@uagm.edu \\ José Reyes, Ana G. Méndez University,jreyes66@email.uagm.edu
}

\begin{abstract}
The objective of this study was to link the different types of APIs with the functionality that companies use to create operationalize functionality and interaction with others. It was analyzed with sets of APIs and various companies from www.programmableweb.com. Through software such as Excel, Node XL, Tableau, visualizations, node analysis, and cluster analysis were performed. The results indicate that certain specialized mashups such as Mapping make concurrent use of the most primitive API resources of different parent companies such as Google and Microsoft, creating a diverse and interconnected development ecosystem. The entertainment and multimedia branches seem to dominate the use of APIs, since only the YouTube API (Google), the Photos API (Flickr), the Music API (Last.fm), and the Telephony API (Twilio) represent more than half of the aggregate use of APIs on the network. On the other hand, eCommerce and Digital Marketing have a significant weight being able to observe a growing development of the APIs of Amazon. The leadership that a parent company can obtain in the development of useful and robust APIs will position it in the most abundant and deep vein of the data mine, which in the end will become the vital information for making strategic decisions by part of any company. APIs and mashups are essential for web development within the API Internet economy.
\end{abstract}

Keywords: APIs, Code, IT, Internet Economy, Mashup

\section{Introduction}

APIs, or application program interfaces, are important tools for business services in all industries. APIs allow the capabilities of one computer program to be used by another. They are a means by which two different programs can communicate. APIs allow companies to grow their businesses quickly. Similar to the Internet and the Web, APIs are driving growth in service-sharing innovation. Organizations in all industries are looking to learn more about APIs and their potential to transform business processes (Glow Touch, 2021).

The API ecosystem paradigm serves as a central point to address the difficulties associated or inherent to the offspring of new technologies and developmental changes within the Internet economy. Businesses in a digitally intensive world operate within ecosystems where their digital business strategy cannot be conceived outside said environment (Bharadwaj, Sawy, Pavlou and Venkatraman, 2013). Under these conditions, firms should consider the role of network effects and multisided business models, where complex and dynamic coordination across multiple companies is required to create and capture value in this digital. 


\section{Issues in Information Systems}

Volume 22, Issue 1, pp. 255-261, 2021

APIs are the fundamental components of digital transformation. Succeeding in today's application economy involves connecting products to customer needs, customers to experiences, applications to devices, organizations to their ecosystem partners. APIs make these connections fast, accurate, and secure (Reyes, 2018).

\section{APIs and Mashup Technology}

The API infrastructure is divided by public release (APIs of diverse functionalities given by major companies such as Google and Facebook), partners, and proprietary software developed for internal use within the private sector (Boyd, 2017). Ecosystems represent a future in which companies leverage their core strengths while using other providers' assets to drive growth (Jhingran, 2018). The developer community assets that must be in place are to first clear terms of service for the API, engage in proper documentation, code snippets, and code for a sample application, create a self-serve API registration, responsive error messages in place, maintain an API specification format, a sandbox environment and finally an API uptime status page (Boyd, 2017). The steps the most successful companies follow when implementing an API strategy according to Digital McKinsey's report (2017) are identification, prioritizing its value, managing monetization actively, create centralized governance and organizational model and drive usage and adoption to gain scale standing.

\section{APIs Categories}

APIs categories demonstrating higher tendencies are video, photos, eCommerce, and telephony which are inclined towards the user experience type, under Google, Flickr, Amazon, and Twilio. APIs instruments that are driving the developers are YouTube, Flickr, Amazon Product Advertising, and Last.fm, inclined towards the user experience type, under the companies Google, Flickr, Amazon, and Last.fm (Reyes, 2018).

\section{SDKs and APIs Categories}

The most common SDKs and APIs categories are photos, social, eCommerce, music, video, and reference; for developers and APIs categories are video, photos, eCommerce, search, music, social, reference, and mapping. APIs and mashup variables share the same categories and order (Reyes, 2018).

\section{API Benefits}

APIs facilitate digital services through the addition of new features and effectively improve B2B interfacing with the website and mobile applications. Besides, the API ecosystem is the new frontier for capturing new revenues, a reduced cost in customer acquisition, and the benefit of saving time when making the APIs available to the market (Wordline Corporate, 2018). Rob van den Dam (2017) provided statistics indicating that $90 \%$ of Expedia revenues derive from APIs, higher than eBays' $60 \%$ and the sales force is $50 \%$, opening a myriad of opportunities for the public and private sector to generate income.

\section{Internet Economy}

The growth in the volume of data, the speed of processes, and data management have allowed companies to innovate by offering their products and services in a personalized and sophisticated way in the digital economy. APIs are the engine that drives the digital economy. All companies in the world are going digital to provide digital services. These digital services need APIs for communication between 


\section{Issues in Information Systems}

Volume 22, Issue 1, pp. 255-261, 2021

companies. Two strategies have been fundamental: open codes and the development of APIs (Forbes, 2020).

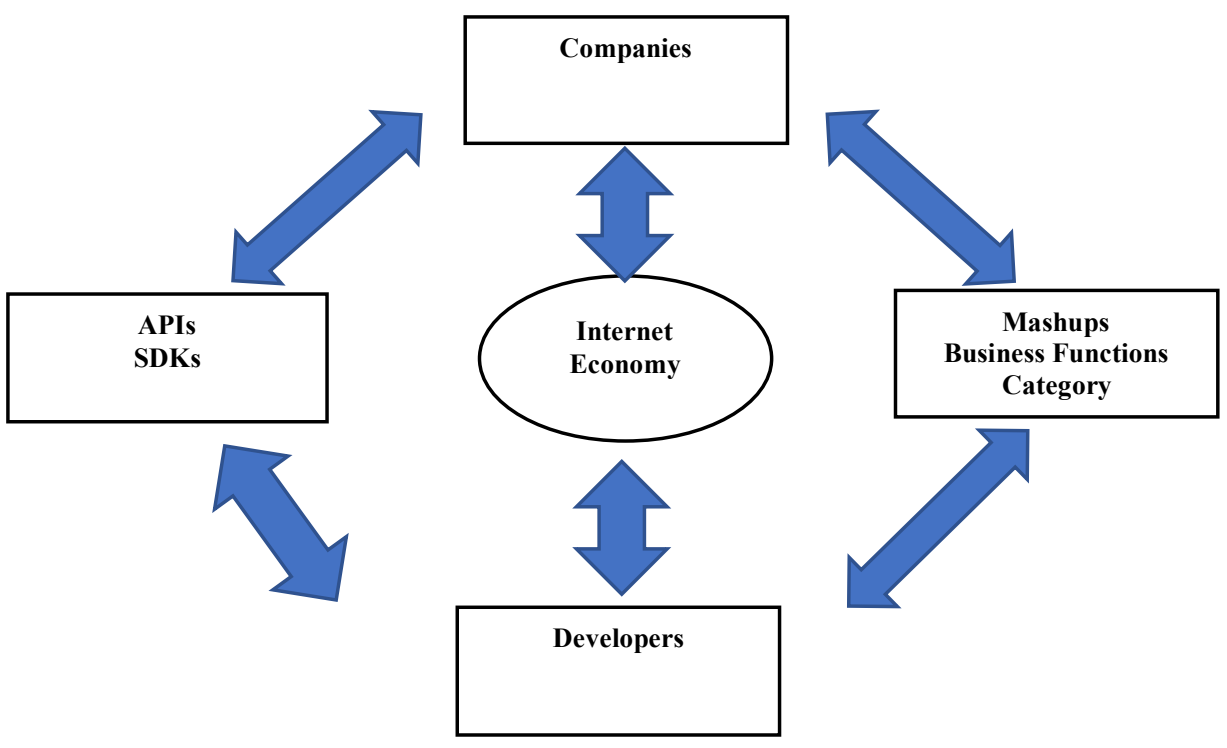

Figure 1. Research framework (Reyes, 2018)

\section{Research methodology}

The Mixed-Method Research framework was used by incorporating secondary data in tabulated, crosstabulation, and standardized form. The data were collected from public databases and available through www.programmableweb.com. The factors studied were: In the curating process, the data was configurated into fields where repeated items were deleted. Cluster analysis was performed using the Excel Node XL template to determine if any grouping existed within the acquired data. For each data group, the vertices and edges were defined to connect the network structures for the visualization process. The Harel-Koren Fast Multiscale and Grid layouts were used to display the results. The data were treated using Cluster Analysis to obtain several summaries and graphics for evidencing a particular relationship between APIs, Parents Companies, and Developers.

\section{Results}

In Figure 2, the development of APIs is dominated by technological giants such as Google, Amazon, Microsoft, and other companies in the field of technology. The entertainment and multimedia branches seem to dominate the use of APIs, since only the YouTube API (Google), the Photos API (Flickr), the Music API (Last.fm), and the Telephony API (Twilio) represent more than half of the aggregate use of APIs on the network. On the other hand, eCommerce and Digital Marketing have a significant weight being able to observe a growing development of the APIs of Amazon, which is the largest online retailer in the world, followed by platforms such as eBay who have developed and improved their API of Product Search. Events such as pandemics and restrictions on free traffic have promoted all the APIs that are focused on the development of eCommerce; thus, the strongest line of APIs of the Amazon giant is oriented Product Advertising and eCommerce. Finally, in a set of APIs with a lower percentage of use, we find services such as search engines (Bing and Google), social networks (LinkedIn), and Education Support such as Wikipedia and Bookmarks. 


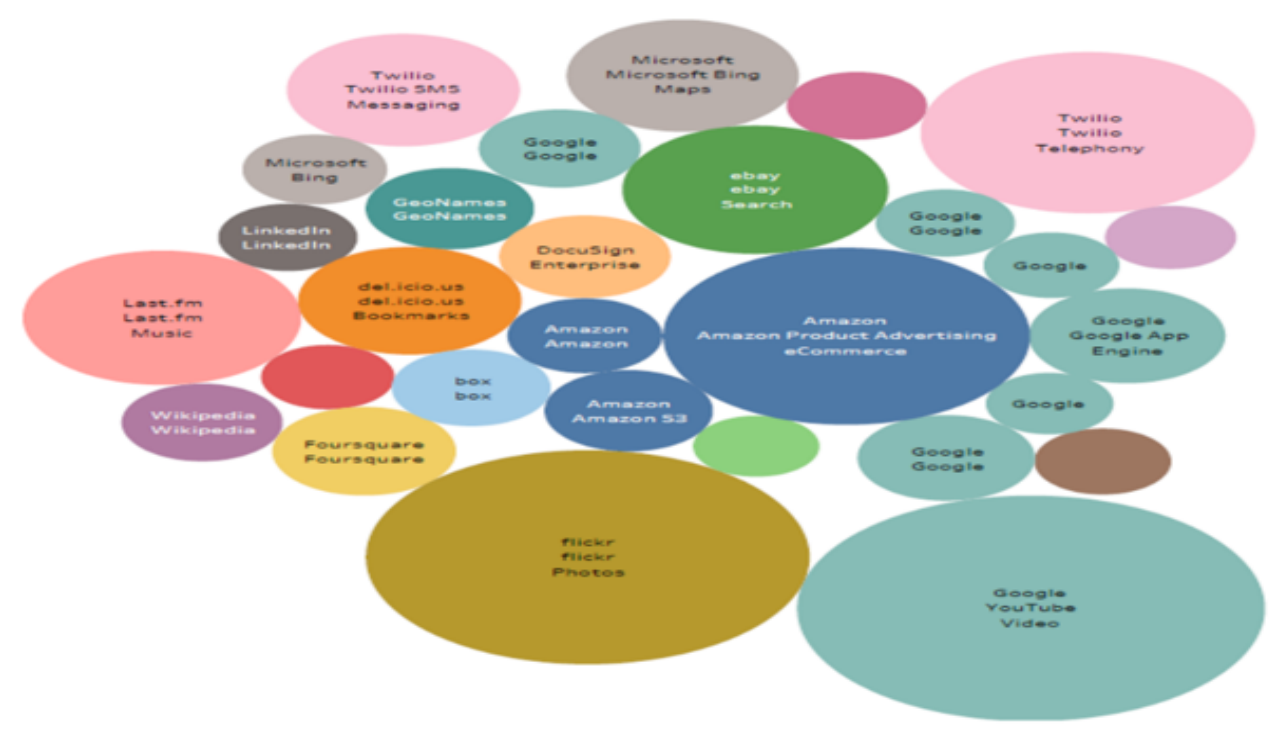

Figure 2. Parent Companies and APIs

In Figure 3, a complex network of relationships between the APIs of the different parent companies is observed. It is very interesting to observe how certain specialized mashups such as Mapping make concurrent use of the most primitive API resources of different parent companies such as Google and Microsoft, creating a diverse and interconnected development ecosystem. This type of "natural" associations allows the development of a self-regulated and interdependent market where large monopolies and the absolute dominance of a single API provider are avoided. It can also be seen that the development of APIs is not accidental but depends on the positioning strategies of companies, which seek to have a presence in different stages of complex business processes, such as eCommerce, Storage, and Cloud, giving companies such as Amazon a definite competitive advantage. In a world where information has become the most precious asset, having a presence in most of the stages of data management processes is necessary for the survival of any company.

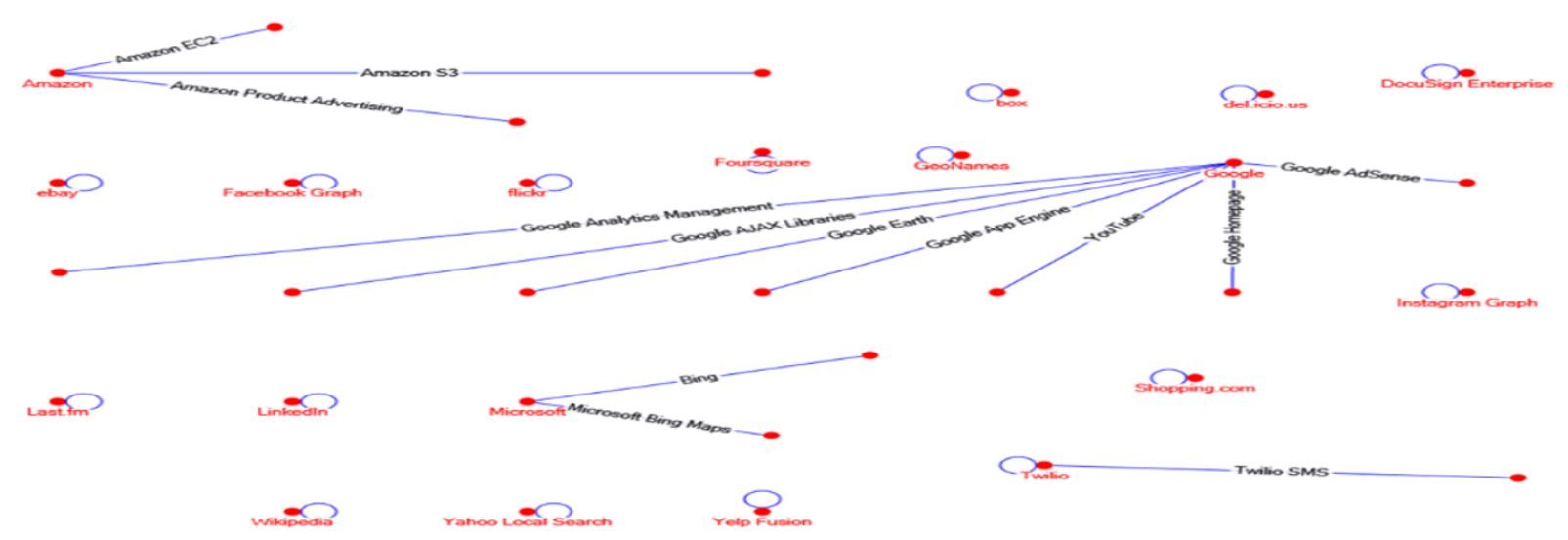

Figure 3. Parent Companies and APIs

In Figure 4, the company that implements the most APIs categories is Google with video, library, tools, analytics, and mapping. Another company with the largest number of APIs categories implemented is 


\section{Issues in Information Systems}

Volume 22, Issue 1, pp. 255-261, 2021

Amazon with shopping, cloud, and storage. The APIs categories that have the most adoption links are mapping and search. These APIs were implemented by Google, Microsoft, and eBay companies. The second category of APIS that has the most adoption links are social. These APIS were implemented by the companies Facebook, Linked In, and Foursquare.

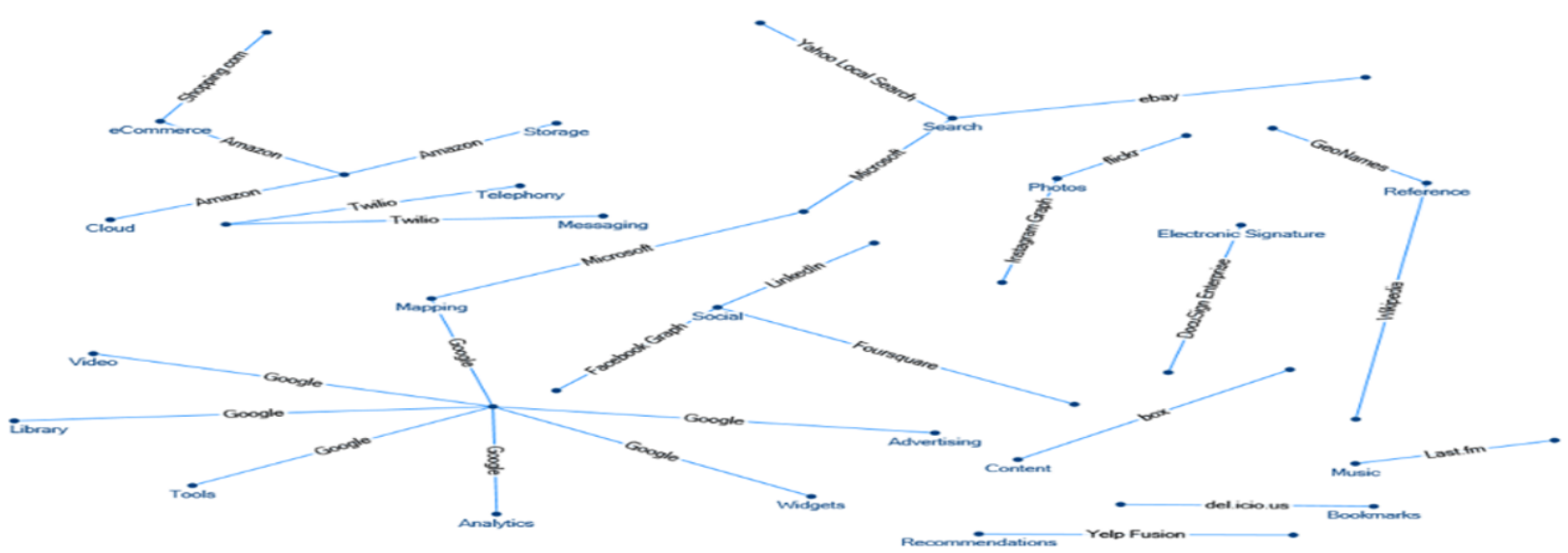

Figure 4. Categories and Parent Companies

In Figure 5, the companies, and APIs categories with the highest use. The category of video APIs was the most outstanding in the usefulness of use with the Google company. The second category of photo APIs was the most highlighted by Flickr. The third category of advertising APIs was the most highlighted by Amazon. The fourth category of music APIs was the most prominent by the Last.fm and the fifth category of search APIs was the most prominent by the eBay company.

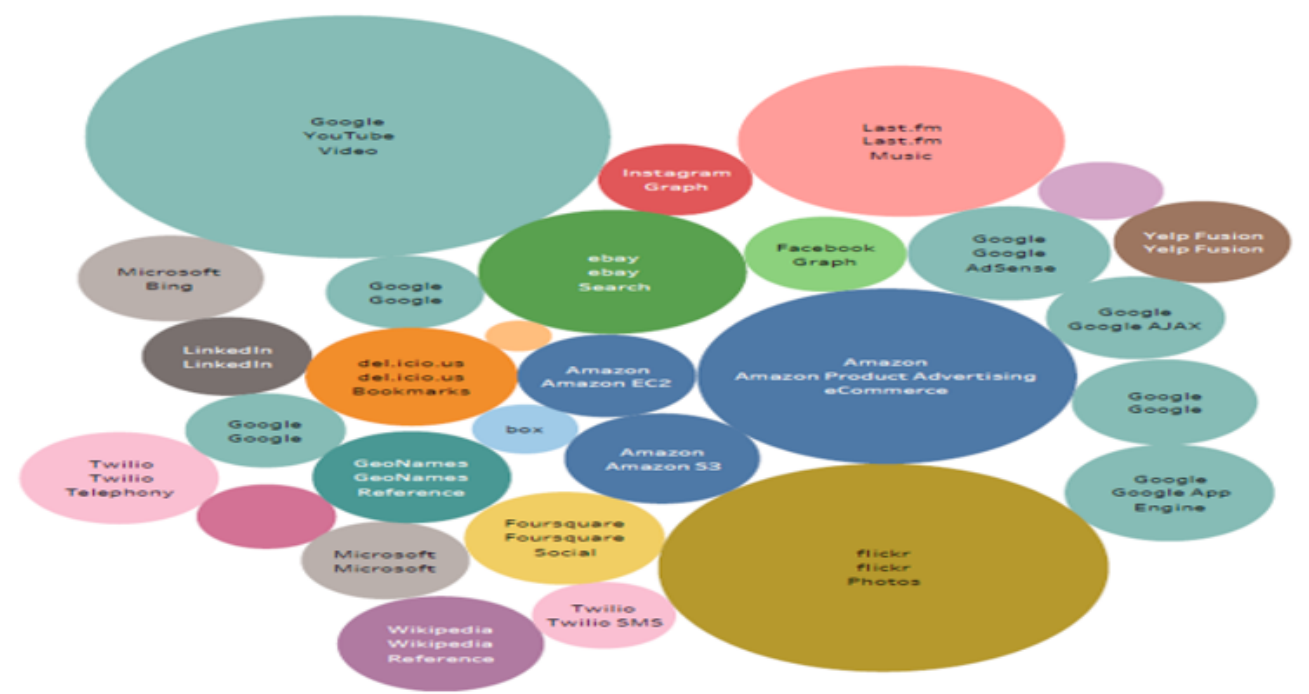

Figure 5. APIs by Company/Category and Developers 


\section{Issues in Information Systems}

Volume 22, Issue 1, pp. 255-261, 2021

\section{Summary}

The leadership that a parent company can obtain in the development of useful and robust APIs will position it in the most abundant and deep vein of the data mine, which in the end will become the vital information for making strategic decisions by part of any company.

Offering useful and attractive services, in the form of APIs, to users is the best strategy for capturing relevant information by any company that wants to guarantee its long-term survival and development. The analysis of the graphs presented, with their respective updates over time, allows governments and regulators to create a notice of the possible formation of certain conglomerates that may eventually become monopolies. It also allows small companies to know the strongest trends and movements in the computer services market, allowing them to move easily towards those market segments that may have more sustained growth soon.

A healthy practice for application developers that require the services of external APIs is the diversity of providers, avoiding at all costs to depend on a single parent company that may eventually stop their operations or prohibits the use of their APIs in certain applications. In this way, a balance of power is maintained in the management and control of applications.

APIs and mashups are essential for web development within the API Internet economy and are traced down to the developer's role and connection with its purpose and functionality. Business entities part take as API service providers as well as product generators to address the demands and needs of the public within the Internet economic model.

\section{References}

Abratt, R., Nel D., \& Higgs, S. N. (1992). An examination of the ethical beliefs of managers using selected scenarios in a cross-cultural environment. Journal of Business Ethics, 11(1), 29-35.

Bharadwaj, A., El Sawy, O. A., Pavlou, P. A., \& Venkatraman, N. (2013). Digital Business Strategy: Toward A Next Generation of Insights. Mis Quarterly, 37(2), 471-482.

Boyd, M. (2017). How to Build Great API Developer Communities Retrieved from https://www.programmableweb.com/news/how-to-build-great-api-developer communities/analysis/2017/09/27

Forbes (2020). The Digital Economy Is the API Economy - And Kong Is King, Forbes.

Glow Touch, (2021). The Importance of APIs for Business. https://www.glowtouch.com/importance-apisbusiness/

Glow Touch Networks Asia, (2017). Study: The majority of HK organizations recognize the importance of APIs. Networks Asia; Newton.

Jhingran, A. (2018). How and Why to Transform Your Business into a Digital Ecosystem API University Retrieved from https://www.programmableweb.com/news/how-and-why-to-transform-yourbusiness-digital-ecosystem/analysis/2018/01/11 


\section{Issues in Information Systems}

Volume 22, Issue 1, pp. 255-261, 2021

RM Van Dam (2017, June 5). Digital Trust and the API economy. https://dtw.tmforum.org/wpcontent/uploads/2017/05/5.-Rob-van-den-Dam-1.pdf

Iyengar, K, Khanna, S., Ramadath, S. and Stephens, D. (2017). What it really takes to capture the value of APIs. Digital Mckinsey.

Reyes, J., (2018) The Importance of Application Programming Interface (API) and Mashups in Web Development for Business Opportunities. 